observations also revealed that pores are still present in the center of many granules. No features were revealed when operating in visible light.

These results demonstrated that the liquid immersion method using IR exposure leads to significant improvement in detecting defects, although the minimum size of detectable features is $1 \mu \mathrm{m}$. However, the researchers indicate that this is not a problem because the size of fabrication defects in this type of ceramic is usually larger.

SIARI S. SOSA

\section{Photopolymerizable Glass Outperforms Traditional Holographic Materials}

Studies of organically modified silica glass have produced a material with high refractive index modulation, high optical quality, and photosensitivity. Plus, the material can be cast as a monolith or a film. In the March 12 issue of Applied Physics Letters, Pavel Cheben of the National Research Council of Canada and Maria L. Calvo of the Complutense University of Madrid have reported that by combining a titanocene photoinitiator and an acrylic monomer with a sol-gel precursor, a material is produced which is ideal for volume holographic recording.

Photoinitiation of ethylene glycol phenyl ether acrylate was accomplished with bis $\left(\mu^{5}-2,4\right.$-cyclopentadien-1-yl)-bis-[2,6difluoro-3-(1H-pyrrol-1-yl)phenyl]titanium. Both photoinitiator and monomer were added to the silica sol prior to the gelation point in concentrations of $0.4-0.8$ $\mathrm{wt} \%$ and $40 \mathrm{wt} \%$, respectively. The solution was stirred for $20 \mathrm{~min}$ at $40^{\circ} \mathrm{C}$ until homogeneous, at which point it was cast into Teflon vials and allowed to gel and dry for 10 days at $60^{\circ} \mathrm{C}$. Thin films of $\sim 260 \mu \mathrm{m}$ thickness were prepared by casting $0.1 \mathrm{~mL}$ of the solution onto a borosilicate glass plate and dried for 5 days at $60^{\circ} \mathrm{C}$.

Characterization of the holographic performance of the glass was achieved by writing a grating pattern into the monolith or thin film, followed by reading the pattern with another beam. The glass refractive index was measured as $n=1.47$ by a prism-coupling technique. The diffraction efficiency dependence on the angle of incidence was measured by detuning the readout beam from the Bragg condition, yielding the grating angular selectivity. The refractive index modulation $(\Delta n)$ amplitude and effective thickness of the grating were calculated using Kogelnik's coupled-wave theory. The gratings in the monoliths produced $\Delta n=1.35 E^{-3}$ for $E \sim 1.5 \mathrm{~J} / \mathrm{cm}^{2}$, and thus a sensitivity of $S=8.6 E^{-4} \mathrm{~cm}^{2} / \mathrm{J}$ ( $E$ is exposure). A dynamic sensitivity $S^{*}=d(\Delta n) / d E$
$=1.8 E^{-3} \mathrm{~cm}^{2} / \mathrm{J}$ was achieved in the region of exposure $0.1-0.35 \mathrm{~J} / \mathrm{cm}^{2}$. The gratings in the $260-\mu \mathrm{m}$-thick films produced $\Delta n=$ $4.5 E^{-3}$ for $E=0.72 \mathrm{~J} / \mathrm{cm}^{2}$ and thus a sensitivity of $S=6.2 E^{-3} \mathrm{~cm}^{2} / \mathrm{J}$.

The researchers discussed the highly efficient formation of gratings in this material in terms of photopolymerization in the light regions. As the monomer is depleted in the light regions, mechanisms involving diffusion and capillary forces move monomers from the dark regions into the light regions where they participate in the photopolymerization process.

The hybrid organic-inorganic glass studied here exceeds the performance of traditional holographic materials. Furthermore, the ability to be cast as a monolith or as a thin film, the porosity of the final glass, the rigid silicon oxide backbone, and no requirement for solvent processing overcomes some problems associated with traditional holographic materials. These combined advantages demonstrate excellent prospects for practical holographic storage devices using this material.

DONALD F. CARTER

\section{Alumina With $50 \%$ Porosity Obtained After Freeze-Drying a Water-Based Slurry}

Ceramic materials with a large surface area are commonly used as catalysts, filters, chemical sensors, and for other similar applications. Freeze-drying is a versatile fabrication method for this type of material and several variants of its use are found in the literature. A group of researchers from the Synergy Ceramics Laboratory and the National Industrial Research Institute of Nagoya in Japan are developing a freeze-dry process starting from a water-based slurry. They maintain that their method is more environmentfriendly since it eliminates the necessity for the use of a binder. Furthermore, the resultant material has good mechanical strength and the pore distribution is easily controlled. In the January issue of the Journal of the American Ceramics Society, these researchers explain how they obtained such a porous structure. They started by mixing alumina powders and a small amount of a dispersant into slurry with distilled water; ball milling occurred

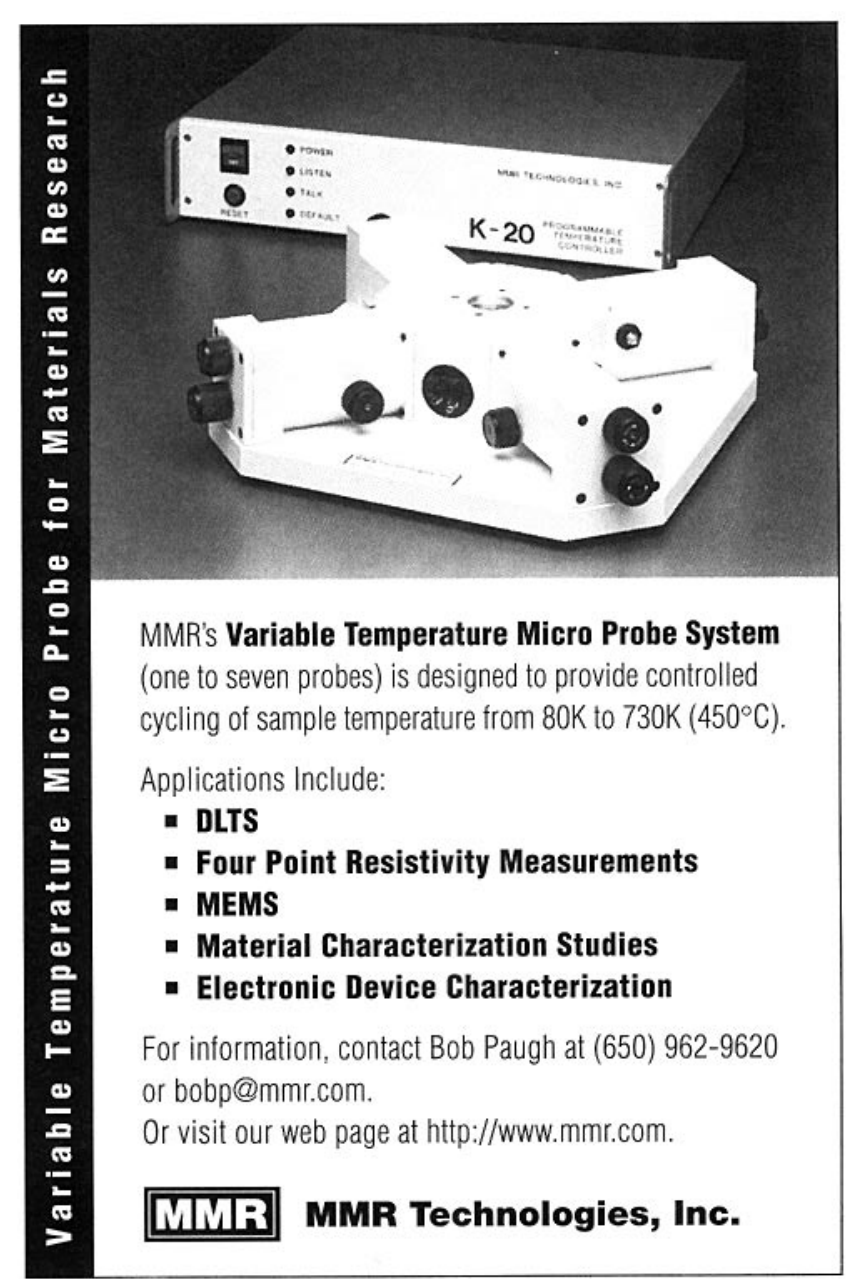

Circle No. 23 on Inside Back Cover 